\title{
Hypervigilance as Predictor of Postoperative Acute Pain: Its Predictive Potency Compared With Experimental Pain Sensitivity, Cortisol Reactivity, and Affective State
}

\author{
Stefan Lautenbacher, PhD, ${ }^{*}$ Claudia Huber, PhD, ${ }^{+}$Miriam Kunz, PhD, + + Andreas Parthum, PhD, ${ }^{+}$ \\ Peter G. Weber, MD, $\S$ Norbert Griessinger, MD, $\dagger$ and Reinhard Sittl, MD $\dagger$
}

\begin{abstract}
Objectives: Pain hypervigilance - a strong attentional bias toward pain - is thought to accompany chronic pain and modulate pain management. Its usefulness as predisposing factor for the development and maintenance of pain has been discussed. The aim of our study was to demonstrate the predictive power of hypervigilance for the development of acute postoperative pain.
\end{abstract}

Methods: Fifty-four young male patients were assessed 1 day before surgery (correction of chest malformation) on a range of psychologic predictors. These predictors included the assessment of hypervigilance (questionnaires as the Pain Catastrophizing Scale, Pain Anxiety Symptom Scale, the Pain Vigilance and Awareness Questionnaire, and the dot-probe task) and affective state, experimental pain sensitivity, and cortisol reactivity. Acute postoperative pain was assessed by ratings of pain intensity 1 week postsurgery and through the amount of analgesics [patientcontrolled epidural analgesia (PCEA)] requested during the first days after surgery.

Results: Pain intensity was significantly explained $(17 \%$ explained variance) by hypervigilance, whereas PCEA performance was not $(10 \%)$. Adding all other predictors led to a significant increase of explained variance $(35 \%)$ for pain ratings and a nonsignificant increase $(19 \%)$ for PCEA. A more parsimonious solution with only heat pain threshold added led to a significant increase in explained variance $(30 \%)$ for pain intensity. Hypervigilance was only moderately correlated with the other predictors.

Discussion: Hypervigilance proved to be a powerful predictor of subjective acute postoperative pain, but was less useful with regard to the amount of requested analgesics. The overlap with other psychologic predictors (affective state, experimental pain sensitivity, and cortisol reactivity) is sufficiently small to consider hypervigilance a promising supplement in psychologic predictor research.

Key Words: hypervigilance, postoperative pain, predictor

(Clin J Pain 2009;25:92-100)

Posto a ostoperative pain in its acute form is an unavoidable aspect of surgery, which requires intensive analgesic treatment. Although management of acute postoperative

Received for publication November 27, 2007; revised May 16, 2008; accepted June 29, 2008.

From the *Department of Physiological Psychology, Otto-Friedrich University Bamberg; †Department of Anaesthesiology, Pain Center; §Department of Pediatric Surgery, Friedrich-Alexander University Erlangen, Germany; and \$Department of Dentistry, Université de Montréal, Canada.

Supported by a research grant of the Deutsche Forschungsgemeinschaft (La 685/6).

Reprints: Prof Stefan Lautenbacher, Physiological Psychology, University of Bamberg, Markuspl. 3, Bamberg 96045, Germany (e-mail: stefan.lautenbacher@uni-bamberg.de).

Copyright (C) 2009 by Lippincott Williams \& Wilkins pain has become largely efficient, outcome is still variable between patients. In the face of similar tissue lesions, some patients report high levels of acute pain despite reasonable dosage of analgesics, whereas other patients do not. ${ }^{1}$ Better prediction of the short-term course of postoperative pain would not only allow for more efficient management of postoperative pain, but would also enrich our understanding of the multiple factors involved in pain genesis. Alongside somatic factors, psychologic factors also have been shown to contribute significantly to postoperative pain. ${ }^{2,3}$ When reviewing the psychologic factors that have been examined so far, it becomes apparent that they tend to be of theoretical distance to the criterion postoperative pain. For example, depression and neuroticism have commonly been studied. 2,3 These are complex psychopathologic or personality constructs, which have been defined as common denominator of behavior, emotion, and cognition in many diverse situations. An alternative approach would be to identify relevant predictors of postoperative pain using specific and circumscribed features of pain processing that are directly related to postoperative pain.

"Hypervigilance" has recently been proposed as predisposing psychologic factor for the development of chronic functional pain like fibromyalgia, chronic back pain, and related disorders. ${ }^{4-7}$ Hypervigilance has seemed also to act as a modulator of outcome in pain management. ${ }^{8}$ Hypervigilance is defined here as the repetitive and automatic prioritization of pain, with the original function of escape and avoidance from physical threat. However, as soon as pain starts to engage attention, disengagement from pain requires strong cognitive efforts. Hypervigilance can also become rigid and maladaptive ${ }^{9,10}$ Hypervigilance can take a conscious and explicit form with individuals being aware that they are monitoring pain. This explicit form of hypervigilance can be assessed using questionnaires. However, the focus of attention is also regulated by processes not accessible to selfobservation, in that they are automatic and implicit. Attention tests like the visual dot-probe $\operatorname{task}^{11}$ and the emotional Stroop paradigm ${ }^{12}$ have been developed for assessment of these unconscious forms of hypervigilance. The major aim of the present study was to assess hypervigilance in both forms (explicit and implicit) for usage as predictors of acute postoperative pain.

The detection of new predictors of acute postoperative pain is more likely to be successful when the overlap between known and traditionally used predictors is not too strong. We intended to test what predictive power hypervigilance measures have alone or in combination with more traditional predictors, stemming largely from biopsychologic and psychopathologic domains. In addition to experimental pain sensitivity (heat, cold, and pressure pain 
thresholds and temporal heat pain summation), cortisol reactivity (morning increase in cortisol, cortisol suppression) was entered as a stress measure into our predictor analysis. As frame of reference, we assessed predictors describing the affective state of the patients, which have traditionally been used in this context, namely depression, state anxiety, and somatization. ${ }^{2,3}$

Our focus was on postoperative pain that develops ex novo owing to surgical tissue lesions; has nociceptive, inflammatory, and neuropathic consequences; and where it can be assumed that pain levels are absent or very low before surgery. Cosmetic surgery can fulfil these requirements because there is no underlying pathology causing pain. Here, we focused on the correction of chest malformations because patients are young and do normally not have comorbid pathologies. ${ }^{13}$ Additionally, such malformations do not usually cause functional problems, which might be associated with sensations of discomfort. ${ }^{14,15}$ The surgical correction causes vast lesions of skin, muscle, and bone tissues, which can be sufficient to cause intense and persistent postoperative pain and which allow for predictor analyses on the basis of sufficient interindividual variance in pain.

In sum, we aimed to assess the predictive power of hypervigilance alone or in combination with alternative psychologic predictors (affective state, experimental pain sensitivity, and cortisol reactivity) in explaining individual differences in postoperative pain (subjective and behavioral indicators) owing to cosmetic surgery (correction of chest malformation). We tested the predictive value of these concepts for prognosis of acute postoperative pain (up to $1 \mathrm{wk}$ after surgery). As the impact of predictor research can be limited if there are too many variables, we tried to limit the number of necessary predictors required for good prognosis.

\section{MATERIALS AND METHODS}

\section{Participants}

Fifty-four male patients with congenital malformations of thorax (mostly funnel chest) between the ages of 14 and 33 years (mean age: $18.5 \pm 4.4 \mathrm{y}$ ) participated in this study. Participants were recruited among inpatients of the Department of Pediatric Surgery of the University of Erlangen. This department is specialized in the surgical correction of thorax malformations; patients from all over Germany are treated here. Weber and Hümmer ${ }^{16}$ have described the surgical technique for correction of this thorax malformation, the so-called Erlangen technique of funnel chest correction in detail. Therefore, only a brief description is given here. The lower part of the sternum is freed through an interior incision. Mobilization of the sternum begins with freeing of the xiphisternum. A spring balance is attached to the sternum with a hook; afterwards the sternum is moved into the desired position. The chest wall is then stabilized with a lightweight transsternal metal implant. After placing wound drains, the chest wall is closed. Patients are discharged from the hospital after 7 to 10 days postsurgery. The metal plate is removed after 1 year.

Participants with chronic pain conditions or with previous surgeries were excluded from the study. We also excluded patients with psychologic disorders (current or previous ones) with the exception of depression, panic disorder, agoraphobia, social or specific phobia, generalized anxiety disorder, and somatoform disorder. The rationale for not excluding patients with the latter conditions was to avoid too low scores in this set of predictor variables. ${ }^{2}$ However, no patient met the diagnostic criteria for these conditions. Patients experiencing strong levels of discomfort owing to functional limitations associated with their chest malformations were also excluded.

The study protocol was approved by the ethics committee of the medical faculty of the University of Erlangen. All participants gave written informed consent. In case of not having attained full age, written informed consent was obtained from their parents and written assent was obtained from them.

\section{Materials and Procedure}

There were 2 sessions. The first session took place 1 day before surgery to assess the predictor variables (hypervigilance, experimental pain sensitivity, and affective state); the second session, approximately 1 week after surgery, was scheduled for assessment of the criterion variables (postoperative pain). The predictor variable cortisol reactivity and the criterion variable patientcontrolled epidural analgesia (PCEA) were assessed outside sessions as described below.

The assessment of the predictor variables in session 1 took place between 4.00 and 8.00 PM. The session, which lasted for approximately 2 hours and 15 minutes, included screening for psychologic disorders by use of a standardized psychologic interview Mini-DIPS [The Mini-DIPS is a structured interview according to Diagnostic and Statistical Manual of Mental Disorders-IV and International Classification of Diseases-10 criteria for current $(6 \mathrm{mo})$ psychologic disorders and covers the following disorders: anxiety, affective, somatization, obsessive-compulsive, posttraumatic stress, acute stress, dissociative, eating disorders, and schizophrenic psychoses]; approximately $30 \mathrm{~min}^{17}$, assessment of hypervigilance (dot-probe task, questionnaires; approximately $1 \mathrm{~h}$ ), assessment of experimental pain sensitivity (pressure and thermal pain; approximately $30 \mathrm{~min}$ ), and assessment of affective state (questionnaires; approximately $20 \mathrm{~min}$ ). Cortisol reactivity was also assessed. Participants were asked to collect salivary samples for 2 consecutive days before admission to the hospital. Postoperative pain (criterion variable) was assessed by 2 measures. First, we used the self-report ratings, which were obtained in session 2 (1 wk postsurgery). Second, we assessed the average amount of requested analgesics (PCEA) during the days postsurgery. Table 1 gives an overview of all predictor and criterion variables assessed.

\section{Assessment of the Predictor Variables}

\section{Assessment of Hypervigilance}

An implicit assessment of hypervigilance was made using a selective attention task for pain-related stimuli (dotprobe task) and an explicit assessment made from questionnaires (German versions of the Pain Catastrophizing Scale (PCS), ${ }^{18}$ Pain Anxiety Symptom Scale (PASS), ${ }^{19}$ and the Pain Vigilance and Awareness Questionnaire $\left.(\mathrm{PVAQ})^{20}\right)$.

Dot-probe Task (Implicit Form of Hypervigilance). The selective attention task for pain-related stimuli used in the current study was based on the dot-probe task described by Keogh et al. ${ }^{11}$ It contains 3 emotional word categories: pain-related (eg, stechend/stinging), social threat (eg, 
TABLE 1. Descriptive Statistics [Mean (SD)] of All Predictor and Criterion Variables

\begin{tabular}{|c|c|c|}
\hline Predictor Variables & Mean & SD \\
\hline \multicolumn{3}{|l|}{ Hypervigilance implicit dot-probe } \\
\hline Pain-related words (index score) & -1.19 & 55.15 \\
\hline Social threat words (index score) & 4.59 & 34.53 \\
\hline Positive words (index score) & -4.67 & 49.24 \\
\hline \multicolumn{3}{|l|}{ Hypervigilance explicit questionnaires } \\
\hline PCS (sum score) & 17.07 & 7.15 \\
\hline PASS (sum score) & 75.02 & 27.79 \\
\hline PVAQ (sum score) & 32.09 & 12.33 \\
\hline \multicolumn{3}{|l|}{ Experimental pain sensitivity } \\
\hline Pressure pain threshold (kPa) & 249.21 & 106.85 \\
\hline Cold pain threshold $\left({ }^{\circ} \mathrm{C}\right)$ & 18.82 & 8.53 \\
\hline Heat pain threshold $\left({ }^{\circ} \mathrm{C}\right)$ & 42.84 & 2.60 \\
\hline Temporal summation (rating scale) & -0.37 & 1.36 \\
\hline \multicolumn{3}{|l|}{ Affective state } \\
\hline SOMS (somatization severity index) & 9.44 & 7.12 \\
\hline STAI (sum score) & 44.08 & 9.98 \\
\hline CES-D (sum score) & 9.98 & 5.28 \\
\hline \multicolumn{3}{|l|}{ Cortisol reactivity } \\
\hline Morning cortisol (ng/mL/min) & 394.69 & 129.81 \\
\hline Cortisol suppression (ng/mL/min) & 201.63 & 120.35 \\
\hline \multicolumn{3}{|l|}{ Criterion variables } \\
\hline $\begin{array}{l}\text { Self-report of postoperative pain } \\
\text { (numerical scale) }\end{array}$ & 3.58 & 1.91 \\
\hline PCEA (boluses requested per hour) & 1.34 & 1.38 \\
\hline
\end{tabular}

CES-D indicates Center for Epidemiological Studies Depression Scale; PASS, Pain Anxiety Symptom Scale; PCEA, patient-controlled epidural analgesia; PCS, Pain Catastrophizing Scale; PVAQ, Pain Vigilance and Awareness Questionnaire; SOMS, Screening of Somatoform Symptoms; STAI, State Trait Anxiety Inventory.

beschämt/ashamed), and positive words (eg, glücklich/ lucky). During visual presentation, these words are paired with neutral words (anstrich/paintwork); neutral-neutral word pairs served as filler items. We translated the words of the original version by Keogh et $\mathrm{al}^{11}$ into German. As not all words fulfilled the criteria of being similar in length and frequency of use, a series of words had to be replaced. In a pilot study, we tested whether each word of the new list (containing more items than necessary) was representative of the designated word category. If this was not the case, these words were excluded from the final use in the dotprobe task.

Following Keogh et al, ${ }^{11}$ a fixation cross was presented in the center of a computer screen for $500 \mathrm{~ms}$. Then, 2 words (a neutral one paired with an emotional one) were presented, 1 below and 1 above the fixation cross for another $500 \mathrm{~ms}$. After this, a dot appeared at the location of 1 of the 2 words. Participants were required to indicate as quickly as possible, by pressing 1 of 2 keys (below and above), where the dot had appeared. A reaction time measurement was taken. After 20 practice trials, participants had to complete 128 test trials (32 trials per word-pair category), all of which were presented in a random order by the computer. Bias indices were calculated on the basis of reaction times to assess separately the attentional bias toward each emotional word category (for more details, see Keogh et $\mathrm{al}^{11}$ ). A positive score indicates an attentional preference for the location of the emotional word, which may suggest vigilance, whereas a negative score may suggest avoidance.

Additionally, patients completed a word-comprehension (Intelligence-Structure-Test $2000 \mathrm{R}$ Form $\mathrm{A}^{21}$ ) and a reading task (analog to the dot-probe task, word pairs of real and nonsense words were presented at a computer screen for $500 \mathrm{~ms}$ and participants had to indicate by key press where the real word appeared) to ensure the patient's capacity to read and understand words quickly enough.

Questionnaires (Explicit Form of Hypervigilance). We tried to assess the more explicit form of hypervigilance using different questionnaires, namely the PCS, ${ }^{18}$ PASS,${ }^{19}$ and the PVAQ. ${ }^{20}$ Whereas the PVAQ seems to be prototypical for this type of measure, PCS and PASS might seem more related to emotional than to cognitive features of pain processing. However, our rationale for using these 3 scales to assess hypervigilance stems from evidence that hypervigilance is an attentional style being enhanced in conditions of fearavoidance and threat and from findings of substantial and significant correlations between these questionnaires. ${ }^{22-25}$ By including these 3 scales to assess the explicit form of hypervigilance, we aimed at using a multimethod approach.

The $\mathrm{PCS}^{18}$ was developed as a measure of catastrophizing related to pain. It contains 13 items that can be divided into 3 subscales, namely rumination, magnification, and helplessness. The items are rated on a 5-point scale. For further analyses, we used the combined sum score of the PCS.

The PASS ${ }^{19}$ is composed of 4 subscales - cognitive anxiety, escape/avoidance, fearful appraisal, and physiologic anxiety - and is designed to measure fear of pain across cognitive, behavioral, and physiologic domains. The items are rated on a 6-point scale. For further analyses, we used the combined sum score (40 items) of the PASS.

The $\mathrm{PVAQ}^{20}$ was developed as a comprehensive measure of attention to pain and has been validated for use in chronic pain and nonclinical samples. ${ }^{26}$ It consists of 16 items that are rated on a 6-point scale and that assess awareness, vigilance, preoccupation, and observation of pain. For further analyses, we used the combined sum score of the PVAQ.

With the exception of the PASS (which had been translated into German and validated by Walter et $\mathrm{al}^{27}$ ), we had to translate the other 2 questionnaires into German, using a standard "forward-backward" procedure. Only if the resulting backward English version was very similar to the original version according to the evaluation of an English native speaker, translation accuracy was considered sufficient. The intercorrelation of the 3 German questionnaires ranged between $r=0.502$ and $r=0.741$, which is in accordance with intercorrelations reported in the literature for English and Dutch versions. ${ }^{22-25}$

\section{Assessment of Experimental Pain Sensitivity}

Experimental pain sensitivity was assessed through the measurement of pain thresholds (pressure pain threshold, cold pain threshold, and heat pain threshold) and the temporal summation of heat pain. All pain thresholds were assessed using a method of limits protocol, similar to that used by Lautenbacher et al. ${ }^{28}$ All participants were familiarized with the test procedures, in that they were trained until they understood all procedures and were able to follow the instructions.

Pressure Pain Threshold. The assessment of pressure pain threshold was performed using a hand-held pressure algometer (Somadic Sales AB, Algometer type II, Sweden) with a probe area of $1 \mathrm{~cm}^{2}$. Site of stimulation was the volar site of the right forearm. The pressure was increased from 
$0 \mathrm{kPa}$ at a rate of change of $50 \mathrm{kPa} / \mathrm{s}$ until the participant felt the first pain sensation and pressed a button. There were 5 trials and the threshold was determined as the average of these trials.

Cold and Heat Pain Thresholds. Thermal stimuli were delivered by use of a Peltier-based, computerized thermal stimulator (Medoc TSA-2001; Medoc Ltd, Ramat Yishai, Israel) with a $3 \times 3 \mathrm{~cm}^{2}$ contact probe. Site of stimulation was the volar site of the left forearm, where the contact thermode was attached. For assessment of cold and heat pain thresholds, thermode temperature increased or decreased from a baseline of $32^{\circ} \mathrm{C}$ at a rate of $1^{\circ} \mathrm{C} / \mathrm{s}$ until the participants felt a first pain sensation and responded by pressing a button. Each time they pressed the button, the temperature returned to baseline temperature, which was held constant until the next trial. Five trials each of heat and cold stimulation were presented. Pain thresholds were determined as the average of the 5 trials.

Temporal Summation. For assessment of temporal summation, a train of 10 stimuli was applied with an interstimulus interval of 2.5 seconds $(0.4 \mathrm{~Hz})$ to the volar site of the left forearm. Temporal summation was tested by comparing the sensations evoked by single pulses (assessed first) to sensations evoked by trains of 10 pulses (only the last pulse was rated), which were delivered 60 seconds later. Participants rated their sensation on a numerical rating scale ( 0 to 10 , being labeled with verbal anchors "no pain" to "strongest pain imaginable"). The stimuli were presented relative to the individual heat pain thresholds $\left(3^{\circ} \mathrm{C}\right.$ above threshold $)$. The heat pain stimuli (saw-tooth shape) started at a baseline temperature of $3^{\circ} \mathrm{C}$ below the individual pain threshold and increased with a heating rate of $4^{\circ} \mathrm{C} / \mathrm{s}$. There were 3 runs. Temporal summation was determined as the averaged difference between sensations evoked by single stimuli and trains of stimuli.

\section{Assessment of the Affective State}

The affective state was assessed with 3 different questionnaires, namely the German versions of the Screening of Somatoform Symptoms (SOMS), ${ }^{29}$ the German version of the State Anxiety Inventory (STAI), ${ }^{30}$ and the German version of the Center for Epidemiological Studies Depression Scale (CES-D, German version: ADS). ${ }^{31}$

The SOMS $^{29}$ is a self-rating scale, which assesses 53 organically unexplained physical symptoms. The state version of the SOMS was applied where participants are asked to rate the intensity of each symptom during the last 7 days on a 5-point Likert scale. For further analyses, we used the sum of all items ("somatization severity index").

The STAI-state ${ }^{30}$ is a self-rating scale and contains 20 items that were designed to measure transitory anxiety states - that is, subjective feelings of apprehension, tension, and worry that vary in intensity and fluctuate on the basis of the situation. Items are rated on a 5-point rating scale.

The CES-D ${ }^{31}$ is a self-rating scale that was designed to assess emotional, somatic, and cognitive symptoms of depressive mood during the last week. It contains 20 items that are rated on a 4-point Likert scale.

\section{Assessment of Cortisol Reactivity}

Following the protocol of Pruessner et al, ${ }^{32}$ patients were asked to sample saliva at the time of awakening and $15,30,45$, and 60 minutes immediately before hospital admission, while still at home, for 2 consecutive days.
Participants were required to expectorate 0.5 to $1.0 \mathrm{~mL}$ of saliva into a plastic vial via a short plastic straw (DRG Instruments GmbH, Germany). At 11 PM of the first day, the patients ingested a tablet of $0.5 \mathrm{mg}$ dexamethasone (DEX) (Merck, Darmstadt, Germany). This was carried out to assess the extent of cortisol suppression produced by a small dosage of DEX (Mini-Dexamethasone-SuppressionTest $^{32}$ ). The patients were informed about the necessity of strictly following the time schedule for saliva sampling and to refrain from eating and brushing teeth before saliva collection to obtain valid data. Patients stored saliva samples at home in their freezer and passed them after admission to the hospital, where they were stored in a $-20^{\circ} \mathrm{C}$ freezer until further analyses.

Salivary cortisol was assayed by use of an enzymelinked immunosorbent assay kit (DRG Instruments, Germany). The intra-assay coefficient of variation was $<5.5 \%$ and the corresponding interassay coefficient was $<6.5 \%$. The analytical sensitivity was $1.48 \mathrm{nmol} / \mathrm{L}$. The competitive immunoassay requires 1.5 hours incubation time and shows robust and reproducible performance.

To quantify the cortisol reactivity, 2 indicators were computed for further analyses: (1) morning cortisol increase during the first hour after awakening (area under the cortisol awakening response curve of day 1) and (2) cortisol suppression after DEX (difference between area under the response curve of days 1 and 2).

The Assessment of the Criterion Variables. As criterion variables, we assessed patients' self-report ratings of postoperative pain and the request of analgesics (PCEA).

\section{Self-report of Postoperative Pain}

One week after surgery, patients were asked to rate the average intensity of their pain during the last week on an 11-point numerical rating scale. The numerical rating scale is labeled with the verbal anchors no pain and strongest pain imaginable.

\section{PCEA}

All patients included in the study received thoracic PCEA. Before the induction of general anaesthesia, an epidural catheter was inserted through the interspinous space at Th6/Th7 or Th7/Th8. The proximal end of the catheter was tunnelled subcutaneously. After the induction of general anaesthesia, $0.375 \%$ ropivacain $(0.2 \mathrm{~mL} / \mathrm{kg})$ was given via epidural route. During surgery, alfentanil was administered intravenously. Thirty minutes before the end of the surgery, patients again received ropivacain via epidural route ( $1 / 3$ of their first dose). Additionally, patients either received $0.5 \mathrm{mg} / \mathrm{kg}$ dexketoprofen or $20 \mathrm{mg}$ / $\mathrm{kg}$ paracetamol intravenously.

Postoperative PCEA was provided using a standard PCA pump. The pump was set to deliver $0.2 \%$ ropivacain plus $1.0-\mu \mathrm{g} / \mathrm{mL}$ sufentanil at a basal rate of 6 to $8 \mathrm{~mL} / \mathrm{h}$. The patient could additionally administer a bolus dose of $3 \mathrm{~mL}$, with a lock-out interval of 30 minutes. Nonopioids were available as rescue analgesia on demand.

The epidural catheter was removed after 3 or 4 days postsurgery. The number of requested PCEA boluses (pump calls, including those during lock-out periods) was assessed and recorded as mean PCEA boluses requested per hour.

In 5 cases, the anesthesiologist did not succeed in placing the epidural catheter. These patients were excluded from the study. One patient reported strong pain at rest during the second postoperative day and was treated by an 
additional bolus of $8-\mathrm{mL}$ ropivacain without success. We removed the partly dislocated catheter and switched the patient to an IV morphine pump (bolus $2 \mathrm{mg}$ ). The patient was not excluded from the study and the PCEA performance during the first 2 days was entered into analysis.

\section{Statistical Analysis}

The predictive power of the 5 groups of predictors, hypervigilance in its explicit and its implicit forms, experimental pain sensitivity, affective state, and cortisol reactivity was tested in 3 steps.

\section{Step 1}

The aim of step 1 was to select from each predictor group the variable that contributes most to the prediction of our criterion measures of acute postoperative pain ("best single predictor"). This step seemed necessary to reduce the number of variables to those being critical for prediction and to get rid of nonpredictive variables. Multiple regression analyses were conducted separately for each predictor group [implicit form of hypervigilance (dotprobe task with pain-related, social threat, and positive words), explicit form of hypervigilance (PASS, PCS, and PAVQ), experimental pain sensitivity (pressure, cold, and heat pain thresholds and temporal summation), cortisol reactivity (area under the morning cortisol and cortisol suppression), and the affective state (SOMS, STAI, and CES-D)] and separately for the 2 criterion measures [self-report of postoperative pain and required analgesics (PCEA)]. On the basis of the values of the $\beta$ coefficients, we selected for each predictor group and each criterion the variable that showed the best performance as predictor.

\section{Step 2}

As the evaluation of the predictive power of hypervigilance for acute postoperative pain was the main interest of our study, we computed separately for the 2 criterion measures-regression analyses, entering only the best single predictor (see Step 1) of the implicit and explicit forms of hypervigilance.

\section{Step 3}

The aim of the third step was to evaluate the extent to which the prediction of acute postoperative pain by the hypervigilance parameters (see Step 2) can be significantly improved by adding predictors from the other 3 predictor groups. Therefore, we conducted blockwise multiple regression analyses. We entered the best single predictors of the implicit and explicit form of hypervigilance into the first block of the regression analyses. Into the second block, we entered the best single predictors of the other predictor groups (overall approach). Regression analyses were conducted separately for the 2 criterion measures. If adding the variables of block 2 led to a significant change in $R^{2}$, we continued with a more detailed approach. We ranked the best single predictors of the other predictor groups according to their $\beta$ coefficients and conducted multiple regression analyses with these predictors being added on in block 2, 3, and 4 in the determined rank order.

For description of simple relationships, Pearson correlation coefficients were computed. Findings were considered to be statistically significant at $\alpha<0.05$.

\section{RESULTS}

The descriptive statistics for all the predictor variables assessed and the criterion variables are displayed in Table 1.

\section{Step 1 (Selection of Best Single Predictor From Each Predictor Group)}

The results of the multiple regression analyses are depicted in Table 2. As can be seen on the left side of this table, most predictor groups in sum were significantly associated with 1 of the 2 criterion variables. Moreover, the predictive power of the implicit and explicit forms of hypervigilance compared well against the other predictor groups.

As described above, for each predictor group, we proceeded by selecting the variable that showed the highest contribution to the prediction of either self-report of postoperative pain or amount of requested analgesics (PCEA) These variables are listed in Table 2 on the right side (marked by gray shade). The selection was based on the relative size of the $\beta$ coefficients in the respective multiple regression equations. Not all of the best single predictors $(50 \%)$ were significant in their multiple regression analysis.

Table 3 gives an overview of the covariation between these best single predictors. As can be seen, significant correlations were found; however, the percentage of common variance (varying between $0.01 \%$ and $16.57 \%$ ) was small enough to suggest that the predictors were not redundant, but reflect different aspects.

\section{Step 2 (Predictive Value of Hypervigilance Alone)}

Self-report of postoperative pain was significantly predicted in a multiple regression analysis by the best single predictors of the implicit and explicit form of hypervigilance $\left(R^{2}=0.167, P=0.012\right)$. However, the predictive value of hypervigilance for the amount of requested analgesics (PCEA) just missed the acceptable level of significance $\left(R^{2}=0.100, P=0.076\right)$.

\section{Step 3 (Augmentation of the Predictive Value by Adding on Other Predictive Variables)}

As can be seen in Table 4, the prediction of the selfreport of postoperative pain significantly increased when the best single predictors of the other predictor groups were added into the regression analyses (significant change in $R^{2}$ ). Next, we examined which of the other variables contributed to the increase of the predictive value. As can be seen in Table 5 , adding "heat pain threshold" led to a significant increase in the prediction of "self-report of postoperative pain"; whereas the inclusion of the best single predictors for "affective state" (somatization) and "cortisol reactivity" (cortisol suppression) did not lead to a significant increase in its predictive value.

With regard to the prediction of PCEA performance, adding the other predictor groups did not lead to a significant increase in $R^{2}$ (Table 4$)$. Thus, we refrained from conducting further analyses.

\section{DISCUSSION}

The aim of the present study was to examine the predictive power of hypervigilance, which is a strong attentional bias toward pain, in the prognosis of acute postoperative pain. Our main findings were as follows: (1) hypervigilance is related to the prognosis of acute postoperative pain. The prediction was significant for subjective 
TABLE 2. Multiple Regression Analyses Between Each Predictor Group and the 2 Criterion Variables and the Selection of "Best Single Predictors" on the Basis of the $\beta$ Coefficients

\begin{tabular}{|c|c|c|c|c|c|}
\hline \multirow[b]{2}{*}{ Predictor Groups } & \multicolumn{2}{|c|}{$R^{2}$} & \multirow[b]{2}{*}{ Single Predictors } & \multicolumn{2}{|c|}{$\beta$ Coefficients } \\
\hline & $\begin{array}{c}\text { Self-report of } \\
\text { Postoperative Pain }\end{array}$ & PCEA & & $\begin{array}{c}\text { Self-report of } \\
\text { Postoperative Pain }\end{array}$ & PCEA \\
\hline \multirow{3}{*}{$\begin{array}{l}\text { Hypervigilance } \\
\text { implicit dot-probe }\end{array}$} & 0.095 & 0.156 & Pain-related words & -0.303 & -0.331 \\
\hline & & & Social threat words & 0.090 & 0.149 \\
\hline & & & Positive words & 0.026 & 0.249 \\
\hline \multirow{3}{*}{$\begin{array}{l}\text { Hypervigilance } \\
\text { explicit } \\
\text { questionnaires }\end{array}$} & 0.142 & 0.053 & PCS & 0.281 & 0.120 \\
\hline & & & PASS & 0.101 & -0.335 \\
\hline & & & PVAQ & 0.029 & 0.269 \\
\hline \multirow[t]{4}{*}{$\begin{array}{l}\text { Experimental pain } \\
\text { sensitivity }\end{array}$} & 0.108 & 0.067 & $\begin{array}{l}\text { Pressure pain } \\
\text { threshold }\end{array}$ & -0.200 & -0.245 \\
\hline & & & Cold pain threshold & -0.211 & -0.014 \\
\hline & & & Heat pain threshold & 0.310 & 0.250 \\
\hline & & & $\begin{array}{l}\text { Temporal pain } \\
\text { summation }\end{array}$ & 0.052 & 0.064 \\
\hline \multirow[t]{3}{*}{ Affective state } & 0.225 & 0.018 & SOMS & 0.294 & 0.090 \\
\hline & & & STAI & 0.175 & -0.136 \\
\hline & & & CES-D & 0.210 & 0.101 \\
\hline \multirow[t]{2}{*}{ Cortisol reactivity } & 0.083 & 0.155 & Morning cortisol & 0.574 & 0.559 \\
\hline & & & Cortisol suppression & -0.626 & -0.795 \\
\hline
\end{tabular}

Significant results are marked as bold, fields shaded in gray represent "best single predictors."

CES-D indicates Center for Epidemiological Studies Depression Scale; PASS, Pain Anxiety Symptom Scale; PCEA, patient-controlled epidural analgesia; PCS, Pain Catastrophizing Scale; PVAQ, Pain Vigilance and Awareness Questionnaire; SOMS, Screening of Somatoform Symptoms; STAI, State Trait Anxiety Inventory.

postoperative pain $(17 \%$ of explained variance using the pain words of the dot-probe task and the PCS) and close to significant for the amount of requested analgesics via PCEA $(10 \%$ of explained variance using the pain words of the dot-probe task and the PASS). (2) Adding other psychologic predictors, which were affective state, experimental pain sensitivity, and cortisol reactivity, leads to a significant improvement in predicting subjective postopera- tive pain $[35 \%$ of explained variance using the hypervigilance parameters plus heat pain threshold, cortisol suppression, and somatoform symptoms (SOMS)]. With regard to the prediction of the amount of requested analgesics via PCEA, the addition of the other predictors led to a near-significant improvement $[19 \%$ of explained variance using the hypervigilance parameters plus heat pain threshold, cortisol suppression, and the Depression Scale (CES-D)]. (3) When a

TABLE 3. Covariation [Correlation Coefficients (r)] Between the "Best Single Predictors"

\begin{tabular}{|c|c|c|c|c|c|c|c|}
\hline & \multirow{2}{*}{$\begin{array}{c}\text { Hypervigilance } \\
\text { (Implicit Form) } \\
\begin{array}{c}\text { Dot-Probe: } \\
\text { Pain } \\
\text { Words* } \dagger\end{array}\end{array}$} & \multicolumn{2}{|c|}{$\begin{array}{l}\text { Hypervigilance } \\
\text { (Explicit Form) }\end{array}$} & \multirow{2}{*}{$\begin{array}{c}\text { Experimental } \\
\text { Pain } \\
\text { Sensitivity } \\
\text { Heat } \\
\text { Pain } \\
\text { Threshold } * \dagger\end{array}$} & \multirow{2}{*}{$\begin{array}{c}\begin{array}{c}\text { Cortisol } \\
\text { Reactivity }\end{array} \\
\text { Suppression* } \dagger \\
\end{array}$} & \multicolumn{2}{|c|}{$\begin{array}{l}\text { Affective } \\
\text { State }\end{array}$} \\
\hline & & $\begin{array}{c}\text { Pain } \\
\text { Catastrophizing } \\
\text { Scale (PCS)* }\end{array}$ & $\begin{array}{c}\text { Pain Anxiety } \\
\text { Symptoms Scale } \\
\text { (PASS) } \dagger\end{array}$ & & & $\begin{array}{l}\text { Somatoform } \\
\text { Symptoms } \\
\text { (SOMS)* }^{\text {SOM }}\end{array}$ & $\begin{array}{c}\text { Depression } \\
\text { Scale } \\
(\text { CES-D) } \dagger\end{array}$ \\
\hline $\begin{array}{l}\text { Pain Words (dot- } \\
\text { probe) }{ }^{* \dagger}\end{array}$ & & $-0.373^{\mathrm{a}}$ & -0.223 & 0.220 & $0.331^{b}$ & -0.161 & -0.068 \\
\hline $\begin{array}{l}\text { Pain } \\
\text { Catastrophizing } \\
\text { Scale (PCS)* }\end{array}$ & & & & 0.015 & -0.108 & $0.409^{c}$ & \\
\hline $\begin{array}{l}\text { Pain Anxiety } \\
\text { Symptoms Scale } \\
(\text { PASS }) \dagger\end{array}$ & & & & 0.007 & -0.112 & & $0.407^{d}$ \\
\hline $\begin{array}{l}\text { Heat Pain } \\
\text { Threshold*† }\end{array}$ & & & & & -0.007 & 0.053 & 0.158 \\
\hline Suppression*† & & & & & & 0.191 & -0.026 \\
\hline $\begin{array}{l}* \text { *Best single pred } \\
+" \text { Best single pred } \\
\text { PCEA indicates p } \\
{ }^{\mathrm{a}} P=0.005 ;{ }^{\mathrm{b}} P=\end{array}$ & $\begin{array}{l}\text { ictors "for self-repor } \\
\text { ictors" for use of } P( \\
\text { atient-controlled epic } \\
.040 ;{ }^{c} P=0.002 ;{ }^{d} P\end{array}$ & $\begin{array}{l}\text { rt of postoperative } \mathrm{p} \\
\text { CEA. } \\
\text { dural analgesia. } \\
\\
=0.001 .\end{array}$ & & & & & \\
\hline
\end{tabular}


TABLE 4. Blockwise Regression Analyses (Overall Approach)_Entering the 2 "Best Single Predictors" of Explicit and Implicit Hypervigilance Into the First Block and the "Best Single Predictors" of the Other Predictor Classes Altogether Into the Second Block of the Regression Analyses

\begin{tabular}{|c|c|c|c|c|c|c|c|}
\hline \multirow[b]{2}{*}{ Criterion Measures } & \multirow[b]{2}{*}{ Step } & \multicolumn{6}{|c|}{ Predictors } \\
\hline & & & $\boldsymbol{R}$ & $P$ & Total $R^{2}$ & $\Delta R^{2}$ & $\Delta P$ \\
\hline \multirow{3}{*}{$\begin{array}{l}\text { Self-report of } \\
\text { postoperative pain }\end{array}$} & 1 & Hypervigilance & 0.408 & 0.012 & 0.167 & & \\
\hline & & $\begin{array}{l}\text { Dot-probe-pain-related words + Pain } \\
\text { Catastrophizing Scale (PCS) }\end{array}$ & & & & & \\
\hline & 2 & $\begin{array}{l}\text { Other predictors } \\
\text { Heat pain threshold + Somatoform } \\
\text { Symptoms (SOMS) + cortisol } \\
\text { suppression }\end{array}$ & 0.587 & 0.001 & 0.345 & 0.178 & 0.011 \\
\hline \multirow[t]{2}{*}{ PCEA } & 1 & $\begin{array}{l}\text { Hypervigilance } \\
\text { Dot-probe-pain-related words + Pain } \\
\text { Anxiety Symptoms Scale (PASS) }\end{array}$ & 0.316 & 0.076 & 0.100 & & \\
\hline & 2 & $\begin{array}{l}\text { Other predictors } \\
\text { Heat pain threshold + Depression Scale } \\
\text { (CES-D) }+ \text { cortisol suppression }\end{array}$ & 0.437 & 0.073 & 0.191 & 0.091 & 0.174 \\
\hline
\end{tabular}

PCEA indicates patient-controlled epidural analgesia.

parsimonious set of predictors of subjective postoperative pain was included, the 2 hypervigilance parameters (dotprobe task with pain words and the PCS) together with the heat pain threshold accounted for $30 \%$ of the explained variance. (4) Hypervigilance parameters seemed to be as adequate for prognosis of postoperative pain as other traditionally used psychologic predictors. They were especially useful in explaining the subjective experience of postoperative pain compared with the behavioral quantification of the amount of requested analgesics (PCEA).

The principal aim of the present study was to investigate whether hypervigilance parameters, alongside other psychologic factors, allow for prognosis of acute postoperative pain. This aim was successful in that our hypervigilance parameters explained as much variance of acute postoperative pain as other psychologic predictors did, both in the current and previous studies. ${ }^{33-35}$ Furthermore, adding other psychologic predictors substantially increased the degree of explained variance, thus suggesting independent predictive power of the hypervigilance parameters. The latter assumption was corroborated by the mainly small correlations between the hypervigilance measures and the other predictors. However, the predictive power of hypervigilance was mainly demonstrated for selfrated intensity of postoperative pain and to a lesser extent for the behavioral indication of postoperative pain, as estimated by the request of analgesics (PCEA). As the subjective intensity of postoperative pain is inferred from the recall of pain over a period of 1 week, we can not exclude the possibility that hypervigilance favorably predicts the recall of pain and not only its experience.

Our intention was also to look for parsimonious sets of predictors. We did this mainly to meet clinical demands on the diagnostic usefulness of predictors. The diagnostic benefit of predicting a small proportion of the variance in postoperative pain by many predictors would be small, and so we tried to identify the best single predictors among a given group of predictors. The PCS and the response bias when pain words were concurrently presented with neutral words in the dot-probe task (explicit and implicit measures of hypervigilance) and the heat pain threshold correlated significantly with the subjective intensity of acute postoperative pain and explained after all $30 \%$ of the variance of postoperative pain. These 3 predictors cover 3 different psychologic domains of processing pain-related information, namely the individual awareness of constantly and anxiously monitoring pain, the automatic engagement of attention when pain-related stimuli are presented concurrently with competing stimuli, and the sensitivity for pain. In the future, it might be promising to look for such powerful combinations of predictors, which are still manageable in diagnostic algorithms.

TABLE 5. Blockwise Regression Analyses (Detailed Approach)_Entering the 2 "Best Single Predictors" of Explicit and Implicit Hypervigilance Into the First Block and "the Best Single Predictors" of the Other Predictor Classes Consecutively Into Block 2, 3, and 4, Ordered According to the Values of Their $\beta$ Coefficients

\begin{tabular}{|c|c|c|c|c|c|c|}
\hline \multirow[b]{2}{*}{ Step } & & \multicolumn{5}{|c|}{ Predictors } \\
\hline & & $\boldsymbol{R}$ & $\boldsymbol{P}$ & Total $R^{2}$ & $\Delta R^{2}$ & $\Delta P$ \\
\hline 1 & Hypervigilance & 0.408 & 0.012 & 0.167 & & \\
\hline & Dot-probe-pain-related words + Pain Catastrophizing Scale (PCS) & & & & & \\
\hline 2 & $\begin{array}{l}\text { Experimental pain sensitivity } \\
\text { Heat pain threshold }\end{array}$ & 0.543 & 0.001 & 0.295 & 0.129 & 0.005 \\
\hline 3 & $\begin{array}{l}\text { Affective state } \\
\text { Somatoform symptoms (SOMS) }\end{array}$ & 0.584 & 0.001 & 0.341 & 0.045 & 0.079 \\
\hline 4 & $\begin{array}{l}\text { Cortisol reactivity } \\
\text { Cortisol suppression }\end{array}$ & 0.587 & 0.001 & 0.345 & 0.004 & 0.583 \\
\hline
\end{tabular}


Interestingly, the implicit measure of hypervigilance, the dot-probe task comparing the attentional bias for painrelated words in competition with neutral words, showed that those patients avoiding the pain-related information were those with the higher acute postoperative pain scores. A similar finding was obtained by Munafo and Stevenson, ${ }^{36}$ who were the first using an implicit measure of attention to predict postoperative pain owing to minor gynecologic surgical procedures by a selective processing task (modified Stroop). At first glance, these findings might seem paradox. However, it has to be kept in mind that our dot-probe task reflects only 1 phase of stimulus evaluation. It might well be that hypervigilant individuals shift attention automatically very briefly toward pain-related information (phase 1) to start attentional avoidance (phase 2) as soon as possible. It is possible that we picked up on the second phase rather than the first. Alternatively, preattentional avoiders of pain information might conduct less elaborate stimulus processing, allowing for more anxiety-related processing of the stimuli. In contrast, those who are vigilant to pain-related information might learn more about the actual threat value of the stimulus and might not activate the fear system unnecessarily. In consequence, such individuals might cope better with pain (eg, Janssen ${ }^{37}$ ). These are quite clearly expost explanations designed to understand our findings and those of Munafo and Stevenson. ${ }^{36}$ A deeper knowledge of the temporal dynamics of attending and avoiding painrelated information as assessed in the dot-probe task is urgently needed.

Our results corroborate our conceptualization of hypervigilance, differentiating between an explicit form (awareness of attentional bias) and an implicit form (unintentional automatic bias of attention), because the 2 forms were not significantly correlated. In partial accord, Asmundson and Hadjistavropoulos ${ }^{38}$ found no relationship between the subjective fear of pain and the dot-probe task performance. Keogh et $\mathrm{al}^{11}$ reported at least 1 significant association between the 2 variables, although this was not strong. Therefore, there seems to be no compelling evidence that refutes our differentiation between explicit and implicit forms of hypervigilance. It should be noted that the situation in which we assessed hypervigilance was special because 1 day before chest surgery, levels of hypervigilance can be expected to be higher than normal.

As state anxiety seems to be a good predictor of acute postoperative pain, the lack of relevance in the present study deserves a comment. ${ }^{2,3,39}$ The variables of the predictor group affective state-which significantly predicted subjective postoperative pain, but not the amount of requested analgesics (PCEA) - were ranked according to their predictive power in the order somatization, depression, and state anxiety. To be fair, the differences between the variables were not huge, and our finding might be unique to the particular patients and surgery under investigation. Although such an assumption cannot be tested here, the present study should provide reason to investigate somatization, that is, the individual presentation of multiple functional body symptoms, further.

A limitation in the explanatory power of our study is that we only examined young men with surgical correction of chest malformations (mainly funnel chest). Although this would seem a good surgical model for psychologic predictor research, it is necessary to replicate our findings in other samples of patients, with other types of surgery, to determine the extent to which our findings can be extrapolated. A comment is also necessary as regards the criteria variables in our study, that is, self-rated postoperative pain and patients' use of the PCEA. The 2 criteria did not span across the same time window in that PCEA use was assessed during the first 3 to 4 days after surgery, whereas the ratings were assessed after 1 week. Early pain ratings, which we also assessed during the first couple of days, were found to be very low both in level and in variance. They apparently reflected mainly the efficiency of analgesic treatment and thus seemed to be secondary to PCEA performance. Therefore, behavioral measures of PCEA seemed to be the early parameters of choice. Whereas self-reported pain, which we assessed after 1 week definitely under psychologically stable conditions in all patients, indicated presumably postoperative pain after the removal of the PCEA protection.

In sum, hypervigilance proved to be a significant predictor of acute postoperative pain, at least as far as the subjective experience is concerned. The overlap with other psychologic predictors (affective state, pain sensitivity, and cortisol reactivity) is sufficiently small to consider hypervigilance a promising supplement in psychologic predictor research. If hypervigilance does prove to be useful in the prediction of other forms of postoperative pain, both in terms of type of surgery (eg, also gynecologic) and the duration of pain (eg, also persistent), it might be worth considering inclusion in a diagnostic algorithm when preparing for surgery.

\section{REFERENCES}

1. Shipton E. Predictors of persistent acute postoperative pain: an opportunity for preventative medicine to reduce the burden of chronic pain. NZ Med J. 2005;118:U1261.

2. Rosenberger PH, Jokl P, Ickovics J. Psychosocial factors and surgical outcomes: an evidence-based literature review. $\mathrm{J} \mathrm{Am}$ Acad Orthop Surg. 2006;14:397-405.

3. Huber C, Lautenbacher S. Relevance of psychological variables for pain after surgery. Anästhesiol Intensivmed. 2008. In press.

4. Crombez G, Eccleston C, Van den Broeck A, et al. Hypervigilance to pain in fibromyalgia: the mediating role of pain intensity and catastrophic thinking about pain. Clin $J$ Pain. 2004;20:98-102.

5. Crombez G, Van Damme S, Eccleston C. Hypervigilance to pain: an experimental and clinical analysis. Pain. 2005;116:4-7.

6. Van Damme S, Crombez G, Eccleston C, et al. Hypervigilance to learned pain signals: a componential analysis. J Pain. 2006;7:346-357.

7. Peters ML, Vlaeyen JW, Kunnen AM. Is pain-related fear a predictor of somatosensory hypervigilance in chronic low back pain patients? Behav Res Ther. 2002;40:85-103.

8. Dehghani M, Sharpe L, Nicholas MK. Modification of attentional biases in chronic pain patients: a preliminary study. Eur J Pain. 2004;8:585-594.

9. Van Damme S, Crombez G, Eccleston C, et al. The role of hypervigilance in the experience of pain. In: Asmundson GJG, Vlaeyen JW, Crombez G, eds. Understanding and Treating the Fear of Pain. Oxford: Oxford University; 2004:71-94.

10. Vangronsveld K, Van Damme S, Peters M, et al. An experimental investigation on attentional interference by threatening fixations of the neck in patients with chronic whiplash syndrome. Pain. 2007;127:121-128.

11. Keogh E, Ellery D, Hunt C, et al. Selective attentional bias for pain-related stimuli amongst pain fearful individuals. Pain. 2001;91:91-100.

12. Roelofs J, Peters ML, Zeegers MP, et al. The modified Stroop paradigm as a measure of selective attention towards 
pain-related stimuli among chronic pain patients: a metaanalysis. Eur J Pain. 2002;6:273-281.

13. Weber PG, Huemmer HP, Reingruber B. Forces to be overcome in correction of pectus excavatum. $J$ Thorac Cardiovasc Surg. 2006;132:1369-1373.

14. Mansour KA, Thourani VH, Odessey EA, et al. Thirty-year experience with repair of pectus deformities in adults. Ann Thorac Surg. 2003;76:391-395.

15. Petersen C. Funnel chest. New aspects since introduction of a minimally invasive surgical technique. Orthopäde. 2003; 32:916-919.

16. Weber PG, Hümmer HP. The new Erlanger technique of funnel chest correction-minimalization of a well working procedure. Zentralbl Chir. 2006;131:493-498.

17. Margraf J. Mini-DIPS. Diagnostisches Kurz-Interview Bei Psychischen Störungen. Berlin: Springer; 1994.

18. Sullivan MJL, Bishop S, Pivik J. The pain catastrophizing scale: development and validation. Psychol Assess. 1995;7: $527-532$.

19. McCracken LM, Zayfert C, Gross RT. The Pain Anxiety Symptoms Scale: development and validation of a scale to measure fear of pain. Pain. 1992;50:67-73.

20. McCracken LM. "Attention" to pain in persons with chronic pain: a behavioral approach. Behav Res Ther. 1997;28:271-284.

21. Amthauer R, Brocke B, Liepmann D, et al. IntelligenzStruktur-Test 2000 R. Göttingen: Hogrefe; 2001.

22. Burns JW, Glenn B, Bruehl S, et al. Cognitive factors influence outcome following multidisciplinary chronic pain treatment: a replication and extension of a cross-lagged panel analysis. Behav Res Ther. 2003;41:1163-1182.

23. Moss-Morris R, Humphrey K, Johnson MH, et al. Patients' perceptions of their pain condition across a multidisciplinary pain management program: do they change and if so does it matter? Clin J Pain. 2007;23:558-564.

24. Roelofs J, Peters ML, McCracken L, et al. The pain vigilance and awareness questionnaire (PVAQ): further psychometric evaluation in fibromyalgia and other chronic pain syndromes. Pain. 2003;101:299-306.

25. Roelofs J, McCracken L, Peters ML, et al. Psychometric evaluation of the Pain Anxiety Symptoms Scale (PASS) in chronic pain patients. J Behav Med. 2004;27:167-183.

26. McWilliams LA, Asmundson GJ. Is there a negative association between anxiety sensitivity and arousal- increasing substances and activities? J Anxiety Disord. 2001;15: 161-170.

27. Walter B, Hampe D, Wild J, et al. Die Erfassung der Angst vor Schmerzen: eine modifizierte deutsche version der Pain Anxiety Symptom Scale (PASS-D). Der Schmerz. 2002; 15(suppl 1):83.

28. Lautenbacher S, Kunz M, Strate P, et al. Age effects on pain thresholds, temporal summation and spatial summation of heat and pressure pain. Pain. 2005;115:410-418.

29. Rief W, Hiller W, Heuser J. SOMS-Das Screening für Somatoforme Störungen (Manual zum Fragebogen). Bern: Huber; 1997.

30. Laux L, Glanzmann P, Schaffner P, et al. Das State-TraitAngstinventar (Testmappe mit Handanweisung, Fragebogen STAI-G Form $X 1$ und Fragebogen STAI-G Form $X$ 2). Weinheim: Beltz; 1981.

31. Hautzinger M, Bailer M. Allgemeine Depressionsskala ADS Weinheim: Belz; 1992.

32. Pruessner JC, Hellhammer DH, Kirschbaum C. Burnout, perceived stress, and cortisol responses to awakening. Psychosom Med. 1999;61:197-204.

33. Cohen L, Fouladi RT, Katz J. Preoperative coping strategies and distress predict postoperative pain and morphine consumption in women undergoing abdominal gynecologic surgery. J Psychosom Res. 2005;58:201-209.

34. den Boer JJ, Oostendorp RA, Beems T, et al. Continued disability and pain after lumbar disc surgery: the role of cognitive-behavioral factors. Pain. 2006;123:45-52.

35. McCarthy SC, Lyons AC, Weinman J, et al. Do expectations influence recovery from oral surgery? An illness representation approach. Psychol Health. 2003;18:109-126.

36. Munafo MR, Stevenson J. Anxiety and surgical recovery. Reinterpreting the literature. $J$ Psychosom Res. 2001;51: 589-596.

37. Janssen SA. Negative affect and sensitization to pain. Scand J Psychol. 2002;43:131-137.

38. Asmundson GJ, Hadjistavropoulos HD. Is high fear of pain associated with attentional biases for pain-related or general threat? A categorical reanalysis. J Pain. 2007;8: $11-18$.

39. Kiecolt-Glaser JK, Page GG, Marucha PT, et al. Psychological influences on surgical recovery. Perspectives from psychoneuroimmunology. Am Psychol. 1998;53:1209-1218. 